\title{
Duality pairs and homomorphisms to oriented and unoriented cycles
}

\author{
Santiago Guzmán-Pro César Hernández-Cruz* \\ Facultad de Ciencias \\ Universidad Nacional Autónoma de México \\ CDMX, México \\ $\{$ sanguzpro, chc\}@ciencias. unam.mx
}

Submitted: Jul 28, 2020; Accepted: Jun 22, 2021; Published: Jul 16, 2021

(C) The authors. Released under the CC BY-ND license (International 4.0).

\begin{abstract}
In the homomorphism order of digraphs, a duality pair is an ordered pair of digraphs $(G, H)$ such that for any digraph, $D, G \rightarrow D$ if and only if $D \not \rightarrow H$. The directed path on $k+1$ vertices together with the transitive tournament on $k$ vertices is a classic example of a duality pair.

In this work, for every undirected cycle $C$ we find an orientation $C_{D}$ and an oriented path $P_{C}$, such that $\left(P_{C}, C_{D}\right)$ is a duality pair. As a consequence we obtain that there is a finite set, $F_{C}$, such that an undirected graph is homomorphic to $C$, if and only if it admits an $F_{C}$-free orientation. As a byproduct of the proposed duality pairs, we show that if $T$ is an oriented tree of height at most 3, one can choose a dual of $T$ of linear size with respect to the size of $T$.
\end{abstract}

Mathematics Subject Classifications: 05C60, 05C75

\section{Introduction}

We consider graphs and digraphs with neither loops nor parallel arcs. For a digraph $D$, we use $V_{D}$ to denote its vertex set and $A_{D}$ to denote its arc set. For undefined terms we refer the reader to [7]. In the homomorphism order of digraphs, a duality pair is an ordered pair of digraphs $(G, H)$ such that for any digraph, $D, G \rightarrow D$ if and only if $D \nrightarrow \rightarrow H$.

Our main result can be considered in three different contexts. We now present a brief introduction to each of them.

The Roy-Gallai-Hasse-Vitaver Theorem [3, 5, 14, 16] states that a graph is $k$-colourable if and only if it admits an orientation with no directed path on more than $k$ vertices. This

\footnotetext{
*Supported by SEP-CONACYT A1-S-8397, CONACYT FORDECYT-PRONACES/39570/2020, and PAPIIT-DGAPA IA104521 grants.
} 
result is a consequence of the fact that a digraph $D$ is homomorphic to the transitive tournament on $k$ vertices, $T T_{k}$, if and only if the directed path on $k+1$ vertices, $\vec{P}_{k+1}$, is not homomorphic to $D$. In terms of duality pairs, $\left(\vec{P}_{k+1}, T T_{k}\right)$ is a duality pair in the homomorphism order of digraphs. In [12] Nešetrril and Tardif proved that if $(A, B)$ is a duality pair in the homomorphism order of digraphs, then $A$ is an oriented tree. Moreover, for any oriented tree, $T$, there is a digraph $D_{T}$ (the dual of $T$ ), such that $\left(T, D_{T}\right.$ ) is a duality pair in the homomorphism order of digraphs. Their result is actually more general, dealing with relational structures, so, as other authors have done, we consider a restriction for the context of this work. In fact, in [11], the same authors consider the problem restricted to digraphs and, for a given oriented tree $T$, they construct a digraph $D_{T}$ such that $\left(T, D_{T}\right)$ is a duality pair. Their construction is simple, but of size exponential on $\left|V_{T}\right|$, raising the following question: Can one choose $D_{T}$ to be of polynomial size with respect to $\left|V_{T}\right|$ ? (Proposed as Problem 1 in [11].) In general, this question has a negative answer [13], but there are some examples where one can pick $D_{T}$ to be of polynomial size with respect to $\left|V_{T}\right|$. For instance, for the family of directed paths, one can choose $D_{T}$ to be the corresponding dual transitive tournament, and thus $D_{T}$ is of linear size when $T$ is a directed path.

Similar notions of duality have been studied also in the context of digraph homomorphisms. In [9], Hell and Zhu defined the class of $B$-cycles as special orientations of cycles, and showed that for a fixed $B$-cycle, $C$, a digraph $D$ is not homomorphic to $C$, if and only if there exists a path $P$ homomorphic to $D$, which is not homomorphic to $C$. They call this notion of duality path duality.

For a set of oriented graphs $F$ the class of $F$-graphs is the class of undirected graphs that admit an $F$-free orientation. In [15], Skrien found a structural characterization for the class of $F$-graphs when $F$ is a set of oriented paths on 3 vertices. Some of these are proper interval graphs, proper circular-arc graphs and comparability graphs. In [4], Skrien's study of $F$-graphs is extended to any set of oriented graphs on 3 vertices. Two of these classes are still lacking a complete structural characterization; the so-called perfectly orientable graphs [15], and the transitive-perfectly orientable graphs [4]. In terms of $F$ graphs, the Roy-Gallai-Vitaver-Hasse Theorem states that, when $F$ is the set of oriented graphs on $k+1$ vertices with a hamiltonian directed path, the class of $F$-graphs is the class of $k$-colourable graphs. In this case, one can assume that such an orientations is also acyclic. The class of graphs that admit an acyclic $F$-free orientation is the class of $F^{*}$-graphs [15]. Another example of such classes are chordal graphs: when $F$ consists of the orientation of the path on 3 vertices such that one vertex has 2 out-neighbours, the class of $F^{*}$-graphs is the class of chordal graphs. This statement follows from the fact that a graph is chordal if and only if it admits a perfect elimination ordering [2].

Even though we mainly deal with duality pairs in the homomorphism order of digraphs, the whole paper is motivated by the study of characterizations of graph classes as $F$ graphs, for a finite set $F$. For each positive integer $n, n \geqslant 3$, we present a finite set of oriented graphs $F_{n}$ such that $F_{n}$-graphs are precisely $C_{n}$-colourable graphs, i.e., graphs that admit a homomorphism to the $n$-cycle. In a way similar to the Roy-Gallai-VitaverHasse Theorem, we use duality pairs as a tool to find such a set $F_{n}$. 
From the viewpoint of oriented cycles and path dualities, it turns out that our result yields another class of cycles, in addition to the $B$-cycles studied in [9], having path duality. The class we propose, $A C$-cycles, is somewhat more restrictive, but the result can be strengthened: for any $A C$-cycle, $C$, we obtain an oriented path $P_{C}$, such that a digraph $D$ is not homomorphic to $C$, if and only if $P_{C}$ is homomorphic to $D$.

The class of $A C$-cycles corresponds to the family of duals, $D_{P}$, for oriented paths $P$ in a special set, for the moment the set $\mathcal{Q}$. Moreover, these $A C$-cycles are duals of linear size with respect to their corresponding path $P$. In [8] Hell and Nešetřil showed that the core of any oriented tree of height 3 is a path in $\mathcal{Q}$. Hence, we conclude that for any tree $T$ of height at most 3 , one can choose a dual $D_{T}$ of linear size with respect to the core of $T$, and thus of linear size with respect to $T$.

The rest of this work is structured as follows. In Section 2, we introduce basic notation, concepts and results needed for later developments. Our main result is stated and proved in Section 3. Finally, in Section 4 we consider the different interpretations of our main result in the three contexts introduced above. Conclusions are briefly presented in Section 5.

\section{Preliminary results}

When $G$ and $H$ are graphs, we write $G \rightarrow H$ to denote that $G$ is homomorphic to $H$. When $x$ and $y$ are vertices of a digraph $D$, we write $x \rightarrow y$ to denote that $(x, y)$ is an arc of $D$. It should always be clear from the context to which interpretation of the symbol $\rightarrow$ we are referring to. Nonetheless, when speaking of homomorphisms, we will use capital letters for digraphs, and when dealing with arcs in a digraph, we will use small-case letter for vertices.

An oriented path $P$ is a sequence of distinct vertices $\left(p_{0}, \ldots, p_{n}\right)$ such that, for each $i \in\{0, \ldots, n-1\}$, either $\left(p_{i}, p_{i+1}\right) \in A_{P}$, or $\left(p_{i+1}, p_{i}\right) \in A_{P}$ (but not both), and $P$ has no more arcs. If $p_{i} \rightarrow p_{i+1}$ we say that $\left(p_{i}, p_{i+1}\right)$ is a forward arc; if $p_{i+1} \rightarrow p_{i}$ the arc $\left(p_{i+1}, p_{i}\right)$ is a backward arc. The direction in which $P$ is traversed is emphasized by saying that the initial vertex of $P$ is $p_{0}$ and the terminal vertex of $P$ is $p_{n}$. If all $\operatorname{arcs}$ in $P$ are forward (backward) arcs, we say that $P$ is a directed path, with forward (backward) direction and denote it by $\vec{P}_{n+1}\left(\overleftarrow{P}_{n+1}\right)$, where $n$ is the number or arcs of $P$. An oriented path is alternating if every two successive arcs are oppositely oriented. We denote by $A_{n}$, the alternating path on $n$ vertices that begins with a forward arc, if $n=1$, then $A_{n}$ denotes the single vertex with no arcs. A semi-walk on a digraph $D$, is a sequence $v_{1} a_{1} v_{2} a_{2} \ldots a_{n-1} v_{n}$, where $v_{i} \in V_{D}$ for $i \in\{1, \ldots, n\}$, and $a_{i}$ is an arc with endpoints $v_{i}$ and $v_{i+1}$, for $i \in\{1, \ldots, n-1\}$. An arc $a_{i}$ in a semi-walk is a forward arc if $a_{i}=\left(v_{i}, v_{i+1}\right)$; otherwise, we say it is a backward arc. A semi-walk is closed if $v_{1}=v_{n}$. The pattern of the semi-walk $v_{1} a_{1} v_{2} a_{2} \ldots v_{n}$, is a sequence $l_{1} \ldots l_{n-1}$ of symbols in $\{\rightarrow, \leftarrow\}$, where $l_{i}=\rightarrow$ if $a_{i}$ is a forward arc; $l_{i}=\leftarrow$ otherwise.

An oriented cycle $C$ is an oriented graph obtained by identifying the initial and terminal vertex of an oriented path $P$. If all arcs have the same direction, we speak of a directed cycle, and denote it by $\vec{C}_{n}$. 
The net length $\ell(X)$ of an oriented path or oriented cycle, $X$, is the number of forward arcs minus the number of backward arcs in $X$, note that the net lentgh may be negative. The following statement if proved in [6], but we use the restatement found in [10] for its simplicity.

Theorem 1. [10] For $n \geqslant 1$, an oriented graph $G$ is homomorphic to $\vec{P}_{n}$, if and only if, every oriented path homomorphic to $G$ has net length at most $n$.

Theorem 1 shows that directed paths have path duality. Now we introduce another family of oriented graphs, proposed by Hell and Zhu in [9], that have path duality. An oriented path $P$ is minimal if it contains no proper oriented path $P^{\prime}$ such that $\ell\left(P^{\prime}\right)=$ $\ell(P)$. An oriented cycle $C=\left(c_{0}, \ldots, c_{n}, \ldots, c_{m-1}, c_{0}\right)$ is a $B$-cycle, if $\left(c_{0}, \ldots, c_{n}\right)$ is a forward directed path, and $\left(c_{0}, c_{m-1}, \ldots, c_{n}\right)$ is a minimal oriented path of net length $n-1$. As mentioned in Section 1, B-cycles have path duality.

Theorem 2. [9] Let $C$ be a B-cycle. A digraph $D$ is homomorphic to $C$ if and only if every oriented path homomorphic to $D$ is also homomorphic to $C$.

A digraph $G$ is balanced if every oriented cycle in $G$ has net length zero. A digraph on $n$ vertices is balanced if and only if $D \rightarrow \vec{P}_{n-1}$ (see [7]). Since every directed cycle has non-null net length, every balanced digraph must be acyclic, and thus there is at least one vertex with no in-neighbours. Let $G$ be a connected balanced digraph and $x \in V_{G}$ such that $d^{-}(x)=0$. We define the level of vertex $v \in V_{G}$ as the net length of any oriented path from $x$ to $v$. The fact that the level of every vertex is well-defined follows from the choice of $G$, i.e. connected and balanced. The maximum level of the vertices in $G$ is called the height of $G$. For two digraphs $G$ and $H$, the interval $[G, H]$ consists on all digraphs $M$ such that $G \rightarrow M \rightarrow H$. The following statement is a useful and well-known result about the homomorphism order of digraphs.

Proposition 3. [8] If $G$ is a balanced digraph of height 3 , then $G \in\left[\vec{P}_{3}, \vec{P}_{4}\right]$.

Two oriented graphs $G$ and $H$ are homomorphically equivalent, if and only if $G \rightarrow H$, and $H \rightarrow G$. Thus, it follows that $G$ and $H$ are homomorphically equivalent, if and only if, for any digraphs $L$ and $R, L \rightarrow G$ if and only if $L \rightarrow H$, and, $G \rightarrow R$ if and only if $H \rightarrow R$. An ordered pair of digraphs $(G, H)$ is a duality pair, if for any digraph $L$, $G \not \rightarrow L$ if and only if $L \rightarrow H$. In this case, we say that $H$ is a dual of $G$. It is not hard to notice that if such a dual exists, then it is unique up to homomorphic equivalence. The transitive tournament on $n$ vertices is denoted by $T T_{n}$. A classic example of a family of duality pairs, is given by the following theorem.

Theorem 4. [1] For $n \geqslant 2$, an oriented graph $G$ is homomorphic to $T T_{n}$ if and only if $\vec{P}_{n+1}$ is not homomorphic to $G$, i.e., for every $n \geqslant 2,\left(\vec{P}_{n+1}, T T_{n}\right)$ is a duality pair.

We conclude this section with the following straightforward observation that we will use more than once in this work.

Observation 5. Let $G, H$ and $R, S$ be pairs of homomorphically equivalent oriented graphs, then $(G, R)$ is a duality pair if and only if $(H, S)$ is a duality pair. 


\section{Main results}

We first define the family of oriented paths for which we will find a family of duals. For $n \geqslant 3$ we denote by $Q_{n}$ the oriented path on $n$ vertices $\left(q_{0}, \ldots, q_{n-1}\right)$ with the following properties: the first two arcs are forward arcs, the suboriented path $\left(q_{1}, \ldots, q_{n-2}\right)$ is an alternating path, and the two final arcs have the same direction. Note that, by the first two conditions, $\left(q_{1}, \ldots, q_{n-k}\right)=A_{n-(k+1)}$ for $1 \leqslant k \leqslant(n-3)$, and $\left(q_{n-3}, q_{n-2}, q_{n-1}\right)=\vec{P}_{3}$ or $\left(q_{n-3}, q_{n-2}, q_{n-1}\right)=\overleftarrow{P}_{3}$, depending on the parity of $n$. This is illustrated in Figure 1 In particular, $Q_{3}$ and $Q_{4}$ are the directed paths on 3 and 4 vertices respectively.
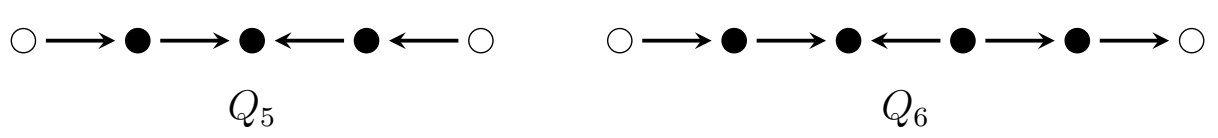

Figure 1: The oriented paths $Q_{n+2}$ with the vertices of their mid-section, $A_{n}$, coloured black $(n \in\{3,4\})$. In $Q_{5}$ the three final vertices induce a directed path with all arcs backward, while in $Q_{6}$ the three final vertices induce a directed path with all arcs forward.

Observation 6. For every integer $n, n \geqslant 5, Q_{n}$ is homomorphic to $Q_{n-2}$. In particular, if $n$ is even then $Q_{n} \rightarrow \vec{P}_{4}$, and if $n$ is odd then $Q_{n} \rightarrow \vec{P}_{3}$.

Proof. Let $n$ be an integer, $n \geqslant 5$, and let $Q_{n}=\left(q_{0}, \ldots, q_{n}\right)$. By identifying $q_{3}$ with $q_{1}$, and $q_{4}$ with $q_{2}$, we obtain a homomorphism from $Q_{n}$ to $Q_{n-2}$.

It is also straightforward to calculate the net length of the oriented paths $Q_{n}$.

Observation 7. For every positive integer $n, n \geqslant 3$, the net length of $Q_{n}$ is described by the equations

$$
\ell\left(Q_{n}\right)= \begin{cases}2 & \text { if } n=3, \\ 3 & \text { if } n \text { is even, } \\ 0 & \text { if } n \text { is odd and greater than } 3 .\end{cases}
$$

It is clear that $\vec{P}_{3}$ maps to $Q_{n}$ for all $n$ at least 3. Now, if $n$ is even, by Observation 7 , $\ell\left(Q_{n}\right)=3$ and since $\ell\left(\vec{P}_{3}\right)=2$, Theorem 1 ensures that $Q_{n}$ does not map to $\vec{P}_{3}$. On the other hand, if $n$ is odd, we know that $Q_{n} \rightarrow \vec{P}_{3}$ by Observation 6 . So the following statement holds.

Lemma 8. For every integer $n, n \geqslant 4, Q_{n}$ is homomorphically equivalent to $\vec{P}_{3}$ if and only if $n$ is odd.

For $n \geqslant 3$, we denote by $A C_{n}$ the oriented cycle obtained from identifying the initial and terminal vertices of the alternating path $A_{n+1}$. For this work we will denote the 


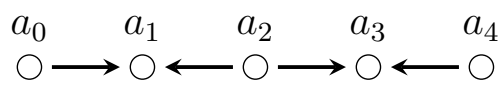

$A_{5}$

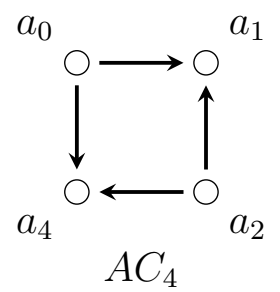

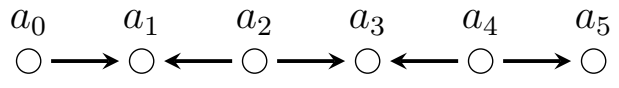

$A_{6}$

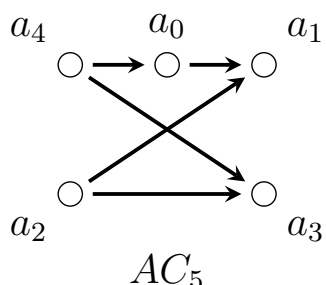

Figure 2: The oriented paths $A_{n+1}$ and oriented cycles $A C_{n}$ for $n \in\{4,5\}$.

vertices of $A C_{n}$ as $\left(a_{0}, a_{1}, \ldots, a_{n-1}, a_{0}\right)$. Note that, if $n$ is even, every two consecutive arcs have opposite direction, and if $n$ is odd, every pair of consecutive arcs, except for $a_{n-1} \rightarrow a_{0} \rightarrow a_{1}$, have opposite direction. In Figure 2 we illustrate $A_{4}, A C_{4}, A_{5}$ and $A C_{5}$.

Lemma 9. For every integer $n, n \geqslant 4$, the cycle $A C_{n}$ is homomorphically equivalent to $\vec{P}_{2}$ if and only if $n$ is even.

Proof. Since $\vec{P}_{2}$ is an asymmetric arc, then $\vec{P}_{2}$ is homomorphic to any non-trivial oriented graph. If $n$ is even, every two consecutive arcs of $A C_{n}$ have opposite direction, thus, the largest directed path of $A C_{n}$ is $\vec{P}_{2}$. But if $n$ is odd, there is a copy of $\vec{P}_{3}$ contained in $A C_{n}$. Therefore, by Theorem $4, A C_{n}$ is homomorphic to $P_{2}$ if and only if $n$ is even.

In order to avoid a very long proof for our main result, we attempt to break it down in a reasonable amount of statements. We start with the following one.

Proposition 10. For every integer $n, n \geqslant 3$, the oriented path $Q_{n+1}$ is not homomorphic to $A C_{n}$.

Proof. First note that when $n=3$ then $A C_{3} \cong T T_{3}$ and $Q_{4} \cong \vec{P}_{4}$. So by Theorem 4 , $Q_{4} \nrightarrow A A C_{3}$. For the remaining cases, i.e., $n \geqslant 4$, we will proceed by contradiction. If $n$ is even, notice that $\vec{P}_{3}$ maps to $Q_{n+1}$. Thus, if $Q_{n+1}$ maps to $A C_{n}$ we obtain a mapping of $\vec{P}_{3}$ to $A C_{n}$. But as $n$ is even, by Lemma $9 A C_{n}$ maps to $\vec{P}_{2}$, and since $\vec{P}_{2} \cong T T_{2}$, this contradicts the fact that $\left(\vec{P}_{3}, T T_{2}\right)$ is a duality pair.

Now suppose that there is an odd integer $n \geqslant 5$ and a homomorphism $\varphi: Q_{n+1} \rightarrow$ $A C_{n}$. We first show that $\varphi$ is not a surjective mapping. Recall that the only vertex in $A C_{n}$ with in-degree and out-degree greater that 0 is $a_{0}$. Note that the only vertices in $Q_{n+1}=\left(q_{0} \ldots q_{n}\right)$ with in-degree and out-degree greater than 0 , are $q_{1}$ and $q_{n-1}$. Thus, $\varphi\left(q_{1}\right)=a_{0}=\varphi\left(q_{n-1}\right)$, and hence $\varphi\left(q_{0}\right)=a_{n-1}=\varphi\left(q_{n-2}\right)$ and $\varphi\left(q_{2}\right)=a_{1}=\varphi\left(q_{n}\right)$. Since $\left|V_{Q_{n}}-\left\{q_{0}, q_{1}, q_{2}, q_{n-2}, q_{n-1}, q_{n}\right\}\right|=n-5$, and $(n-5)+3<n=\left|V_{A C_{n}}\right|, \varphi$ is not surjective. Now we observe that the existence of such a non-surjective homomorphism leads to a contradiction. First, it is not hard to verify that $A C_{n}-a_{i}$ is homomorphic 
to $\vec{P}_{3}$ for any $i \in\{2, \ldots, n-2\}$. Since $\varphi$ is not surjective, and by previous arguments $\left\{a_{n-1}, a_{0}, a_{1}\right\} \subseteq \varphi\left[V_{Q_{n+1}}\right]$, then by composing homomorphisms, $Q_{n+1}$ is homomorphic to $\vec{P}_{3}$. This contradicts the fact that $\ell\left(Q_{n+1}\right)=3$ (Observation 7 ) and Theorem 1 .

Proposition 10 implies that if $D$ is a digraph and $Q_{n+1} \rightarrow D$ then $D \nrightarrow A C_{n}$. The following lemma asserts that the converse implication holds when $n$ is odd.

Lemma 11. For any odd integer $n, n \geqslant 3$, and any digraph $D$, if $Q_{n+1} \nrightarrow \rightarrow D$ then $D \rightarrow A C_{n}$.

Proof. Let $D$ be a digraph such that $Q_{n+1} \not \rightarrow D$ and let $G$ be the underlying graph of $D$. Since our goal is to construct a homomorphism $D \rightarrow A C_{n}$ we may assume that $D$ is connected (and so is $G$ ).

Let $X$ be the set of vertices of $D$ having positive in- and out-degrees. If $X$ is an empty set, then $D \rightarrow T T_{2}$ and so $D$ trivially maps to $A C_{n}$. From now on we will assume that $X$ is a non-empty set. The remaining vertices of $D$ can be partitioned into two sets: the set of vertices $I$ having only in-neighbours and the set of vertices $O$ having only out-neighbours. It is straightforward to verify that $I$ and $O$ are independent sets. Moreover, $X$ is also an independent set; otherwise there is a pair of vertices $x_{1}, x_{2} \in X$ such that $\left(x_{1}, x_{2}\right) \in A_{D}$ and since $x_{1}$ has an in-neighbour $u$ and $x_{2}$ had an out-neighbour $v$, then $\vec{P}_{4} \rightarrow D$. Recall that by Observation $6, Q_{n+1} \rightarrow \vec{P}_{4}$ and thus $Q_{n+1} \rightarrow D$ which is a contradiction. We now refine our partition $(X, I, O)$ with respect to the distance in $G$ of vertices to $X$. For $i \in\{1, \ldots, \operatorname{diam}(G)\}$ we define $I_{i}=\{v \in I: d(v, X)=i\}$ and $O_{i}=\{v \in O: d(v, X)=i\}$.

By construction of the sets $X, I_{i}$ and $O_{i}$ for $i \in\{1, \ldots, \operatorname{diam}(G)\}$, it is not a hard task to verify that for every $\operatorname{arc}(u, v) \in A_{D}$ one of the following statements holds:

- $u \in O_{1}$ and $v \in X$ or $u \in X$ and $v \in I_{1}$,

- there is a positive integer $i$ such that $u \in O_{i}$ and $v \in I_{i-1} \cup I_{i+1}$, or

- there is a positive integer $i$ such that $u \in O_{i}$ and $v \in I_{i}$.

If the third case does not hold, then the overall structure of $D$ can be depicted as follows

$$
\cdots I_{4} \leftarrow O_{3} \rightarrow I_{2} \leftarrow O_{1} \rightarrow X \rightarrow I_{1} \leftarrow O_{2} \rightarrow I_{3} \leftarrow O_{4} \cdots
$$

Noticing that in this case $D \rightarrow \vec{P}_{3}$ is no more than routine work. Since $\vec{P}_{3} \rightarrow A C_{n}$, then $D \rightarrow A C_{n}$.

Now suppose that there is a positive integer $i$ such that there is an arc from $O_{i}$ to $I_{i}$ in $D$, and let $i_{0}$ be the minimum of all such integers. We depict the structure of $D$ with respect to the previously defined partition in Figure 3.

Note that if $i_{0} \geqslant \frac{n-1}{2}$ we can map $D$ to $A C_{n}$. Indeed, first consider the relation $x \sim y$ defined by the following conditions:

- $x, y \in X$,

- $x, y \in I_{i}$ or $x, y \in O_{i}$ for some $i \in\left\{1, \ldots, \frac{n-1}{2}-1\right\}$, or 


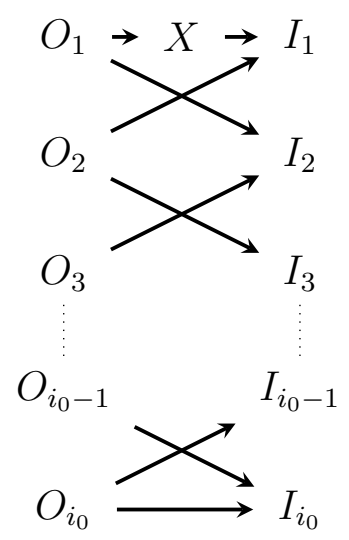

Figure 3: The overall structure of $D$ with respect to the proposed partition.

- $x \in I_{i}$ and $y \in I_{j}$ for some $i, j \in\left\{\frac{n-1}{2}, \ldots, \operatorname{diam}(G)\right\}$ or $x \in O_{i}$ and $y \in O_{j}$ for some $i, j \in\left\{\frac{n-1}{2}, \ldots, \operatorname{diam}(G)\right\}$.

Then, by identifying the vertices in the same $\sim$-equivalence class we obtain an oriented graph isomorphic to $A C_{n}$.

In order to conclude our proof we show that if $i_{0}<\frac{n-1}{2}$, then there is a homomorphism from $Q_{n+1}$ to $D$ which contradicts the choice of $D$, and so the claim of this lemma will follow. Consider an arc $(u, v)$ from $O_{i_{0}}$ to $I_{i_{0}}$. By construction of our partition, there are two alternating paths of length $i_{0}$, one from $u$ to some vertex $x_{u} \in X$ and another one from $v$ to some vertex $x_{v} \in X$. By the choice of $X, x_{u}$ has an in-neighbour $y_{u}$ and $x_{v}$ an out-neighbour $y_{v}$. Since the previously described $u y_{u^{-}}$and $v y_{v}$-paths can be concatenated with the arc $(u, v)$, we obtain a path in $D$ isomorphic to $Q_{2 i_{0}+4}$. Recall that $i_{0} \leqslant \frac{n-1}{2}-1$ so $2 i_{0}+4 \leqslant n-1$ and both quantities are even. Hence, by Observation $6, Q_{n+1}$ maps to $Q_{2 i_{0}+4}$ and thus to $D$. We have reached the anticipated contradiction and thus the lemma follows.

It is straightforward to verify that if a digraph $D$ has a symmetric arc, then every oriented tree is homomorphic to $D$. Also, if $G$ is an oriented graph, then $D$ is not homomorphic to $G$. So if $T$ is an oriented tree, and $D_{T}$ is any of its duals, then $D_{T}$ is an oriented graph, $T \rightarrow D$, and $D \nrightarrow D_{T}$. For this reason, we state and prove the following theorem for oriented graphs only, but clearly it also holds for general digraphs.

Theorem 12. For any integer $n, n \geqslant 3$, an oriented graph $G$ is homomorphic to $A C_{n}$ if and only if $Q_{n}$ is not homomorphic to $G$. In other words, the ordered pair $\left(Q_{n+1}, A C_{n}\right)$ is a duality pair.

Proof. If $n$ is even, by Lemma $8, Q_{n+1}$ is homomorphically equivalent to $\vec{P}_{3}$, and by Lemma $9, A C_{n}$ is homomorphically equivalent to $\vec{P}_{2}$. Thus, by Observation 5 , if $n$ is even, $\left(Q_{n+1}, A C_{n}\right)$ is a duality pair if and only if $\left(\vec{P}_{3}, \vec{P}_{2}\right)$ is a duality pair. The latter 
statement holds since $\vec{P}_{2} \cong T T_{2}$, and $\left(\vec{P}_{3}, T T_{2}\right)$ is duality pair (Theorem 4 ). Finally, if $n$ is odd, by Proposition 10 we know that if $Q_{n+1} \rightarrow D$ then $D \not \rightarrow A C_{n}$. On the other hand, if $Q_{n+1} \nrightarrow \rightarrow D$, by Lemma 11 then $D \rightarrow A C_{n}$. Therefore, $\left(Q_{n+1}, A C_{n}\right)$ is a duality pair for every positive integer $n, n \geqslant 3$.

In Figure 4 we exhibit two of the duality pairs described in Theorem 12.

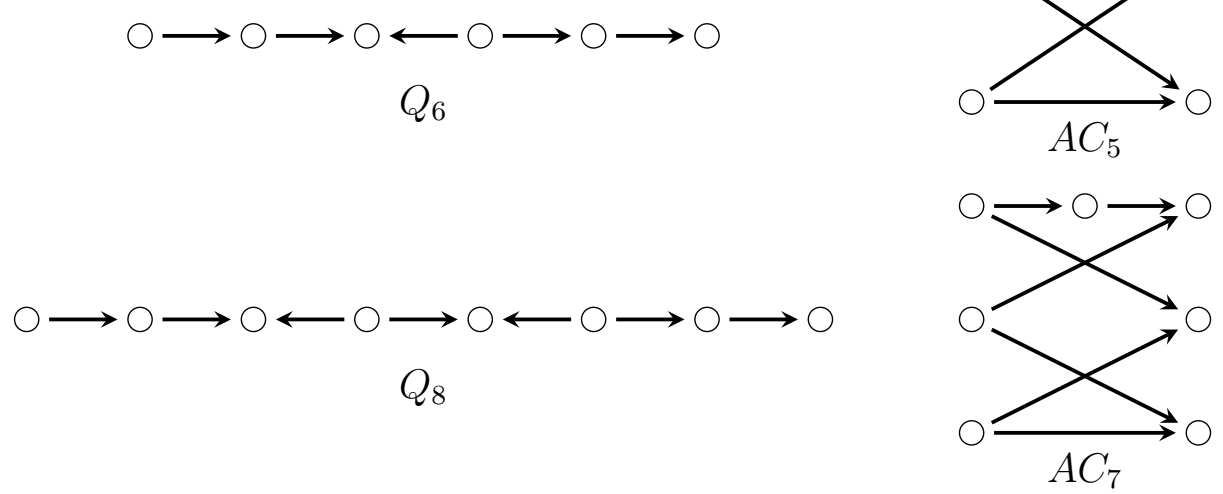

Figure 4: Two duality pairs $\left(Q_{6}, A C_{5}\right)$ and $\left(Q_{8}, A C_{7}\right)$.

\section{Implications}

We say that an oriented cycle $C$ is an $A C$-cycle if $C \cong A C_{n}$ for some positive integer $n$. The following result is a weaker version of Theorem 12 .

Corollary 13. Let $C$ be an $A C$-cycle. $A$ digraph $D$ is homomorphic to $C$, if and only if every oriented path homomorphic to $D$ is also homomorphic to $C$.

Thus, in terms of path dualities, we can extend Theorem 2 with this corollary as follows.

Theorem 14. Any oriented cycle $C$ that is a B-cycle or an $A C$-cycle, has path duality, i.e., a digraph $G$ is homomorphic to $C$, if and only if every path homomorphic to $G$ is also homomorphic to $C$.

Recall that a digraph is a core, if and only if it is not homomorphic to any proper subgraph. The following statement is a well-known result in homomorphism order of digraphs.

Proposition 15. [8] Let $G$ be a digraph in $\left[\vec{P}_{3}, \vec{P}_{4}\right]$, then $G$ is homomorphically equivalent to $Q_{n}$ for some even integer $n \geqslant 4$. Moreover for every even integer, $n \geqslant 4$, the path $Q_{n}$ is a core. 
Now, we give a partial answer to the problem of determining if one can choose a dual, $D_{T}$, of an oriented tree, $T$, of polynomial size with respect to $\left|V_{T}\right|$.

Theorem 16. Let $T$ be an oriented tree of positive height at most 3 , and $P_{T}$ its core. One can choose a dual $D_{T}$ of $T$ of linear size with respect to $\left|V_{P_{T}}\right|$. Since $\left|V_{P_{T}}\right| \leqslant\left|V_{T}\right|$, then $D_{T}$ is of linear size with respect to $\left|V_{T}\right|$.

Proof. If $T_{1}$ is a tree of height 1 , then $\vec{P}_{2}$ is homomorphically equivalent to $T_{1}$. When $T_{2}$ is a tree of height 2 , then $T_{2}$ is homomorphically equivalent to $\vec{P}_{3}$. So by Theorem 4 , $\left(T_{1}, T T_{1}\right)$ and $\left(T_{2}, T T_{2}\right)$ are duality pairs.

If $T_{3}$ is a tree of height 3 , then by Propositions 3 and $15, T_{3}$ is homomorphically equivalent to a path $P$, and $P \cong Q_{n+1}$ for an odd integer $n, n \geqslant 3$. Thus, by Theorem 12 , $\left(T_{3}, A C_{n}\right)$ is a duality pair. For a tree $T$ in any of these cases, the size of the chosen dual is linear with respect to the core of $T$.

Finally, we connect our result to the study of hereditary graph properties characterized as the class of $F$-graphs for a finite set $F$. For $n \geqslant 4$ an even integer, denote by $F_{n}$, the set of surjective homomorphic images of $Q_{n}$. Clearly, $F_{n}$ is a finite set since the order of any oriented graph in $F_{n}$ is bounded by $n$. Notice that the last three results of this section deal with (undirected) graphs.

Theorem 17. Let $G$ be a graph and let $n$ be an even integer. If $n \geqslant 4$, then $G$ is $C_{n-1}$ colourable if and only if $G$ is an $F_{n}$-graph. That is, there is an orientation of $G$ with no induced oriented graph in $F_{n}$.

By Observation 6 , the directed path on 4 vertices belongs to $F_{n}$ for an even integer $n \geqslant 4$. Thus, it is straightforward to notice that the directed 3 - and 4-cycles also belong to $F_{n}$. Thus, from the previous corollary we obtain the following one.

Corollary 18. Let $G$ be a graph and let $n$ be an even integer. If $n \geqslant 4$, then $G$ is $C_{n-1}$ colourable if and only if $G$ is an $\left(F_{n}-\left\{\vec{C}_{3}, \vec{C}_{4}\right\}\right)^{*}$-graph. That is, there is an acyclic orientation of $G$ with no induced oriented graph in $F_{n}-\left\{\vec{C}_{3}, \vec{C}_{4}\right\}$.

In particular, $F_{6}$ consists of the eight oriented graphs depicted in Figure 5.

Corollary 19. The following statements are equivalent for a graph $G$.

- $G$ is homomorphic to the 5-cycle,

- $G$ admits an orientation that has no semi-walk with pattern $\rightarrow \rightarrow \leftarrow \rightarrow \rightarrow$,

- $G$ admits an $\left\{\vec{C}_{3}, T T_{3}, \vec{P}_{4}, \vec{C}_{4}, C_{4}^{\prime}, Q_{6}, D_{5}, \overleftarrow{D}_{5}\right\}$-free orientation, and

- $G$ admits an acyclic $\left\{T T_{3}, \vec{P}_{4}, C_{4}^{\prime}, Q_{6}, D_{5}, \overleftarrow{D}_{5}\right\}$-free orientation 

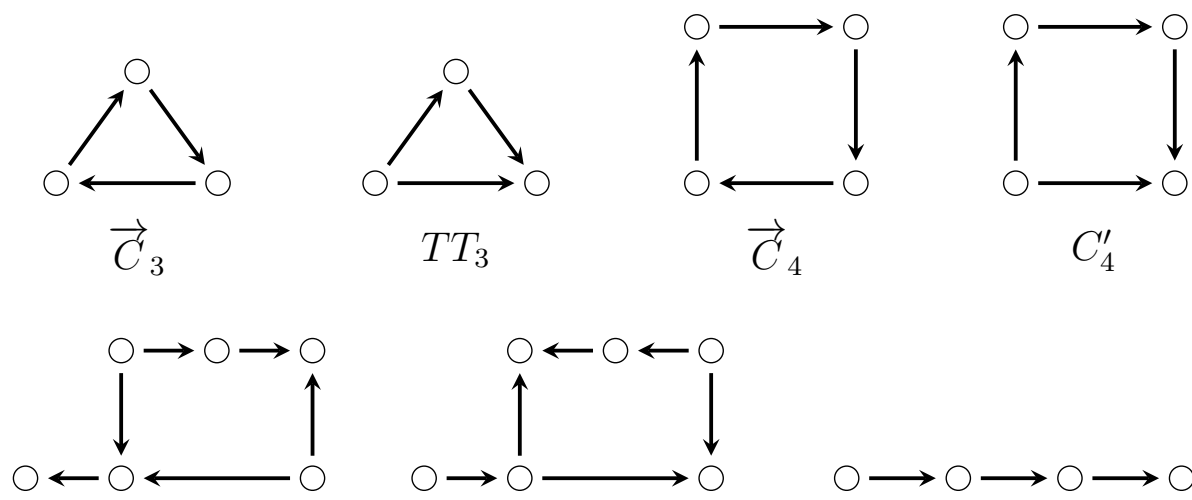

$D_{5}$
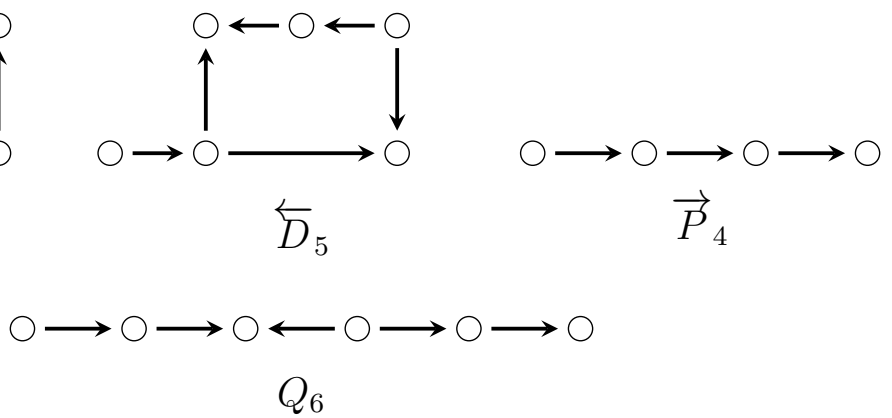

Figure 5: The eight oriented graphs in $F_{6}$.

\section{Conclusions}

Consider an odd integer $n, n \geqslant 5$, and an oriented graph $G$. Note that a polynomialtime certifying algorithm that determines if an oriented graph $G$ is homomorphic to $A C_{n}$ can be obtained from the proof of Lemma 11. The yes-certificate is the refined partition $X,\left\{O_{i}\right\},\left\{I_{i}\right\}$ that induces a homomorphism $\varphi: G \rightarrow A C_{n}$, and the no-certificate is the semi-walk constructed when $i_{0}<\frac{n-1}{2}$.

As a nice consequence, we obtain that graphs admitting a homomorphism to an odd cycle can be characterized as those graphs having an orientation avoiding a well defined finite set of oriented graphs.

As previously mentioned, Nešetřil and Tardif exhibited a family of oriented paths $P_{n}$ such that any dual of $P_{n}$ is of exponential size with respect to $\left|V_{P_{n}}\right|$ [13]. It is not hard to observe that the net length of $P_{n}$ increases as $n$ increases. Theorem 16 shows that for every oriented tree $T$ of height at most 3 there is a dual of polynomial size with respect to $\left|V_{T}\right|$. We finish this work by proposing the following questions.

Question 20. Is it true that for any positive integer $k$, there is a polynomial $p_{k}(x)$ such that for any oriented tree $T$, if $\ell(T) \leqslant k$ then there is a dual $D_{T}$ of $T$ such that its size is bounded by $p_{k}\left(\left|V_{T}\right|\right)$ ?

In the event that the answer to Question 20 results negative, from the results obtained in the present work, the following question still makes sense.

Question 21. Which is the maximum integer $k$, for which one can find a polynomial $p_{k}(x)$ such that for any oriented tree $T$, if $\ell(T) \leqslant k$ then there is a dual $D_{T}$ of $T$ such that its size is bounded by $p_{k}\left(\left|V_{T}\right|\right)$ ? 


\section{Acknowledgements}

We thank the anonymous referees for their very thorough revision of the manuscript; their suggestions helped to greatly improve the quality of the present work.

\section{References}

[1] G. S. Bloom and S. A. Burr, On unavoidable digraphs in orientations of graphs, J. Graph Theory 11(4) (1987) 453-462.

[2] D. R. Fulkerson and O. A. Gross, Incidence matrices and interval graphs, Pacific J. Math. 15(3) (1965) 835-855.

[3] T. Gallai, On directed paths and circuits, Theory of Graphs (Proc. Colloq., Tihany, 1966), Academic Press, New York, 1968, 115-118

[4] S. Guzmán-Pro and C. Hernández-Cruz, Orientations without forbidden patterns on three vertices, arXiv:2003.05605.

[5] M. Hasse, Zur algebraischen Begrundung der Graphentheorie, I, Math. Nachr. 28 (1964/1965) 275-290.

[6] R. Häggkvist, P. Hell, D. J. Miller, and V. Neumann-Lara, On multiplicative graphs and the product conjecture, Combinatorica 8 (1988) 63-74.

[7] P. Hell, and J. Nešetřil, Graphs and Homomorphisms, volume 28 of Oxford Lecture Series in Mathematics and its Applications, Oxford University Press 2004.

[8] P. Hell, and J. Nešetřil, Images of Rigid Digraphs, European J. Combin. 12(1) (1991) $33-42$.

[9] P. Hell, H. Zhou, and X. Zhu, Homomorphisms to Oriented Cycles, Combinatorica 13(4) (1993) 421-433.

[10] P. Hell, X. Zhu, The Existence of Homomorphisms to Oriented Cycles, SIAM J. Discrete Math. 8(2) (1995) 208-222.

[11] J. Nešetřil, and C. Tardif, A dualistic approach to bounding the chromatic number of a graph, European J. Combin. 29 (2008) 254-260.

[12] J. Nešetřil, and C. Tardif, Duality theorems for finite structures (characterizing gaps and good characterizations), J. Combin. Theory Ser. B 80 (2000) 80-97.

[13] J. Nešetřil, and C. Tardif, Short Answers to Exponentially Long Questions: Extremal Aspects of Homomorphism Duality, SIAM J. Discrete Math. 19(4) (2005) 914-920.

[14] B. Roy, Nombre chromatique et plus longs chemins d'un graphe, Rev. Fr. Inform. Rech. Oper. 1 (1967) 129-132.

[15] D. J. Skrien, A relationship between triangulated graphs, comparability graphs, proper interval graphs, proper circular-arc graphs, and nested interval graphs, J. Graph Theory 6(3) (1982) 309-316 
[16] L. M. Vitaver, Determination of minimal colouring of vertices of a graph by means of Boolean powers of the incidence matrix, Dokl. Akad. Nauk SSSR 147 (1962) 758-759 (in Russian).

[17] X. Zhu, A Polynomial Algorithm for Homomorphisms to Oriented Cycles, J. Algorithms 19(3) (1995) 333-345. 